\title{
Practice Spotlight: Marie Craig and The Arthritis Program (TAP) at Southlake Regional Health Centre
}

\author{
Marie Craig, BScPharm \\ The Arthritis Program (TAP) \\ Southlake Regional Health Centre \\ Newmarket, Ontario
}

\footnotetext{
C
} are for patients with arthritis has improved greatly over the past 20 years. As a pharmacist working in rheumatology, Marie Craig (known previously as Marie Chambers) has been an active participant in this evolution. Joining the rheumatology team at Southlake Regional Health Centre, Newmarket, Ontario, in 1986, she has made the transition from educating patients about gold injections in the 1980s to educating them about self-injection of methotrexate in the 1990s and the use of biologics in the new millennium.

Ms Craig started her career in arthritis care after 16 years at St Michael's Hospital in Toronto. Joining the Pharmacy Department of Southlake Regional Health Centre in 1986, she soon became involved with the rheumatology team. In 1991, Southlake received funding from the Ontario Ministry of Health and Long-Term Care to establish a rheumatology outpatient program, officially known as The Arthritis Program or TAP. Through this funding Ms Craig formally joined The Arthritis Program, where her new colleagues became the program's coordinator, 2 occupational therapists, 2 physical therapists, a social worker, and 2 rheumatologists. TAP, which provides care for patients with 135 types of arthritis, not only services the York Region but also draws 2000 new patient referrals per year from a much larger geographic area.

The TAP pharmacist's practice is integrated with the program's vision of central coordination of all health care disciplines during any patient's visit. This centralization of care includes documenting the activities of all disciplines in a single health care record, which contributes to more comprehensive care and reduces duplication of not only charting but also patient visits. The one-site, one-chart (including rheumatologists' notes and health professionals' charting) vision eliminates the need for each discipline to ask the same questions, while offering the patient easy and continuous access to integrated care. Guided by the Registered Health Professions Act, each health care professional is educated experientially in rheumatology and encouraged to fine-tune his or her discipline-specific skills while thinking "outside the box".

Working together at one site in the Tannery Mall in Newmarket, TAP staff members offer arthritis assessment, education, and treatment by individual appointment, group sessions (for inflammatory arthritis, osteoarthritis, fibromyalgia, and osteoporosis), Rheumatology Clinic appointments (with urgent access to the rheumatologist and other members of the team), and telephone consultation. Through interactions with patients, the TAP pharmacist and other team members frequently identify medication issues requiring intervention. The TAP pharmacist intervenes on behalf of the patient either through the Rheumatology Clinic or through an individual appointment for medication review and education. A large component of the TAP pharmacist's practice involves assessing medication tolerance and ensuring appropriate education to prepare patients to self-manage their disease.

This work environment and role allowed Ms Craig to practise according to the philosophy of pharmaceutical care, even before this philosophy had been firmly defined or taught. After 20 years of exposure to patient medication concerns, Ms Craig developed a medication decision model that asks patients to balance the probable side effects of the arthritis (if untreated) against the benefit of the medication and its possible side effects. The medication information is presented to the patient in an unbiased manner, with the ultimate understanding that it is the patient who will make an informed decision about his or her care. At times, the TAP pharmacist acts as a mediator, advocating on behalf of both the patient and the rheumatologist to find a medication management strategy agreeable to both.

Ms Craig and the TAP team document clinical activities in the patient chart and track workload statistics for the 
Ministry of Health and Long-Term Care. This documentation reflects the value and contribution of the pharmacist and has been successfully used to support funding requests for additional TAP pharmacists. Ms Craig believes strongly that pharmacists, like all other team members, should be funded specifically by the program with which they are involved. This helps to ensure that the pharmacist's role will be entrenched in planning any expansion of services.

TAP offers a model of care that is envied by many but duplicated by few. Ms Craig's practice setting has been a highly requested site for many fourth-year Structured Practical Experience Program (SPEP) student pharmacists and PharmD students from the University of Toronto's Faculty of Pharmacy. Looking back on her shift from a primary role of distribution pharmacist to her current role as a care provider within TAP, Ms Craig recommends that all pharmacists immerse themselves in the care of the patients within their practice. This approach is both stimulating and rewarding, as pharmacists develop and apply their professional knowledge and skills to the care of individual patients. At the same time, patients and professionals in other disciplines can observe and experience the important role and benefit of pharmacists in patient care. By giving back to the profession, individual pharmacists will have an impact that extends well beyond their daily practice.

Readers who are interested in learning more about the potential role of the pharmacist in caring for patients with arthritis are encouraged to contact Marie Craig at mcraig@southlakeregional.org.

The Practice Spotlight series highlights the accomplishments of Canadian pharmacists with unique practices in hospitals and related health care settings. If you have a unique or innovative practice, or you know someone else who should be profiled, please submit your contact information to Mary Ensom, Editor of CJHP (cjhpedit@cshp.ca), and one of our Associate Editors will be in touch with you.

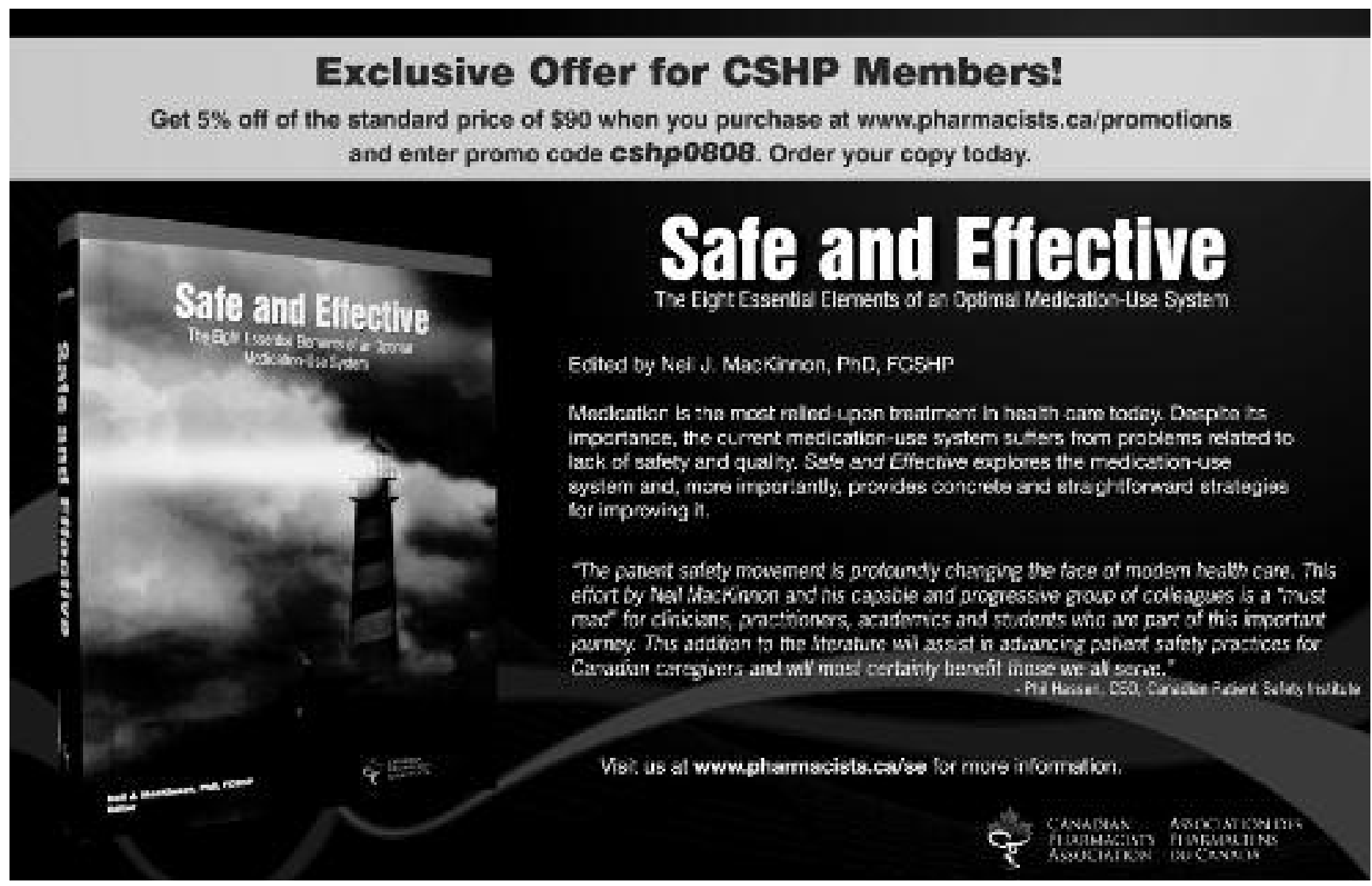

